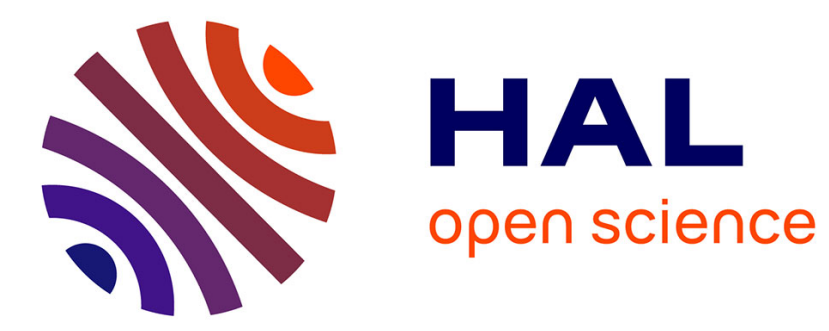

\title{
Enhanced spectral compression in nonlinear optical fibres
}

\author{
Sonia Boscolo, Christophe Finot
}

\section{To cite this version:}

Sonia Boscolo, Christophe Finot. Enhanced spectral compression in nonlinear optical fibres. 18th International Conference on Transparent Optical Network (ICTON 2016), Jul 2016, Trente, Italy. 10.1109/ICTON.2016.7550496 . hal-01312244

\section{HAL Id: hal-01312244 https://hal.science/hal-01312244}

Submitted on 14 Jul 2016

HAL is a multi-disciplinary open access archive for the deposit and dissemination of scientific research documents, whether they are published or not. The documents may come from teaching and research institutions in France or abroad, or from public or private research centers.
L'archive ouverte pluridisciplinaire HAL, est destinée au dépôt et à la diffusion de documents scientifiques de niveau recherche, publiés ou non, émanant des établissements d'enseignement et de recherche français ou étrangers, des laboratoires publics ou privés. 


\title{
Enhanced spectral compression in nonlinear optical fibres
}

\author{
Sonia Boscolo and Christophe Finot* \\ Aston Institute of Photonic Technologies, Birmingham B4 7ET, Royaume-Uni \\ * Laboratoire Interdisciplinaire Carnot Bourgogne, UMR 6303 UBFC-CNRS, 2178 Dijon, France \\ christophe.finot@u-bourgogne.fr
}

\begin{abstract}
We propose two new approaches to enhance the spectral compression process arising from nonlinear pulse propagation in an optical fibre. We numerically show that an additional sinusoidal temporal phase modulation of the pulse enables efficient reduction of the intensity level of side lobes in the spectrum. Another strategy is to select a regime of propagation in which normal group-velocity dispersion reshapes the initial stretched pulse to a near-Fourier-transform-limited rectangular waveform.
\end{abstract}

Keywords: nonlinear fibre optics, spectral compression, nonlinear pulse shaping.

\section{INTRODUCTION}

The effect of self-phase modulation (SPM) on an optical pulse propagating in a nonlinear optical fibre is ordinarily associated with broadening of the pulse spectrum [1]. However, this is not always the case with chirped pulses, where pulses with an initial negative chirp (such as that imparted by an anomalously dispersive element) in the normal group-velocity dispersion (GVD) region can experience spectral narrowing [2, 3]. Indeed, as a result of SPM, the propagating pulse will have the frequencies of the leading edge downshifted and the frequencies of the trailing edge upshifted. Therefore, in the case of an initially negatively chirped pulse, both the trailing and leading edges of the pulse will experience a spectral shift in the direction of the central frequency. It is worth noting here that the anomalous GVD enhances the effect of SPM rather than compensating it. However, in general an input pulse with a negative linear chirp cannot be compressed to the Fourier transform limit, because the cancellation of linear and nonlinear phases cannot in general be made complete. In order to overcome this limitation, several strategies have been proposed and experimentally demonstrated, based on the use of complex phase modulation of the initial pulse [4] or the use of a pre-shaped input pulse profile such as a parabolic waveform [5].

In this paper, we present two new approaches to the enhancement of the performance characteristics of the spectral compression process. First, we numerically show that an additional sinusoidal temporal phase modulation of the pulse enables efficient reduction of the intensity level of spectral side lobes. Then we show that the progressive reshaping of the initial pulse toward a rectangular waveform that results from the combination of SPM and normal GVD facilitates the full compensation of the initial chirp [6, 7].

\section{SPECTRAL COMPRESSION IN THE NONLINEAR REGIME}

First, we briefly recall the results that can be obtained in the nonlinearity-dominant regime of propagation, in which the fibre dispersion is of little importance and, thus, can be neglected. For the purpose of illustration, at the entrance of the fibre we take a Gaussian pulse that has been temporally stretched by a factor of 20 in an anomalously dispersive linear element. We use distance and time variables normalized to $L_{N}=1 /\left(\gamma P_{0}\right)$ and $T_{0}$, respectively, where $P_{0}$ and $T_{0}$ are the peak power and the characteristic temporal value of the pulse at the input to the linear element, and $\gamma$ is the Kerr nonlinearity coefficient of the fibre. The narrowing of the pulse spectrum is quantified with the spectral compression factor $C_{f w h m}\left(C_{r m s}\right)$ defined as the ratio of the spectral full-width at halfmaximum (FWHM) [root-mean-square (rms)] width at some distance in the fibre and the entrance of the fibre. In order to assess the quality of the spectral compression, we use the Strehl ratio $S$ defined as the ratio of the maximum spectral brilliance of the actual pulse to the spectral brilliance obtained assuming a flat temporal phase of the pulse [5].

The longitudinal evolution of the pulse spectrum in Fig. 1(a) clearly shows that significant spectral compression occurs in the fibre, followed by splitting of the spectrum into an increasing number of substructures. At the propagation distance $z_{1}$, where the linear and nonlinear phases cancel each other in the central part of the pulse, the pulse spectrum is effectively conveniently compressed, and the spectral profile does not display any oscillations [Fig. 1(b)]. At the distance of optimum compression in terms of Strehl ratio, $z_{2}$, owing to interference between different pulse parts having the same instantaneous frequency, the spectrum is narrower and features an increased brightness [7], but it suffers from residual side lobes. For further propagation in the fibre, as a result of this interference phenomenon the pulse develops strong and detrimental oscillations in the wings together with strong spectral side lobes, as it appears from the spectrum at the distance $z_{3}$. The evolutions of parameters $S$ and $C$ are given in Fig. 1(c). The moderate values of $S$ and $C_{r m s}$ at $z_{2}$ are a signature of the oscillatory structure of the spectrum. 

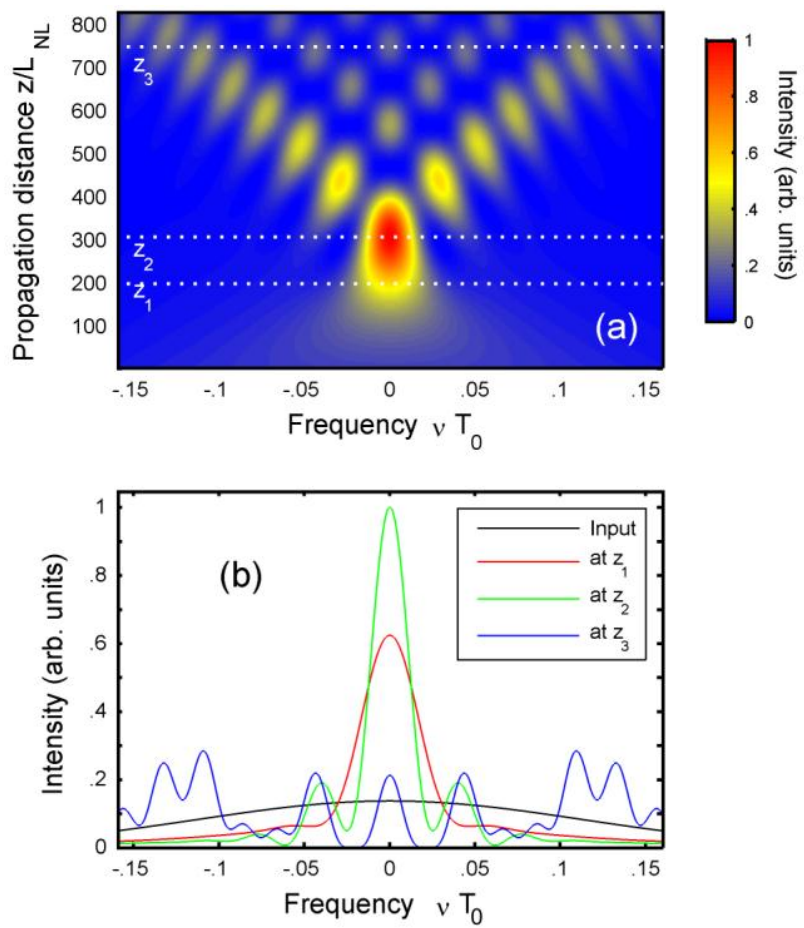

(c)
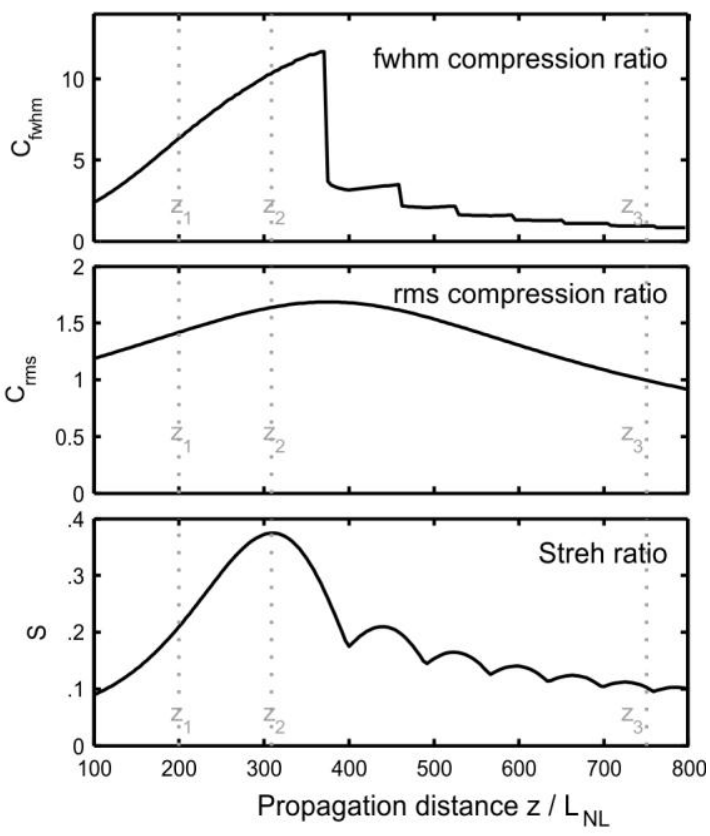

Figure 1. Spectral pulse compression in a purely nonlinear fibre. (a) Longitudinal evolution of the spectral intensity profile. (b) Spectra at different distances in the fibre: the entrance of the fibre (black), the point $z_{1}$ of chirp cancellation near the pulse centre (red), the point $z_{2}$ of maximum Strehl ratio (green), and the point $z_{3}$

(blue). (c) Longitudinal evolutions of the compression ratios $C_{f w h m}, C_{r m s}$ and the Strehl ratio $S$.

\section{USE OF AN ADDITIONNAL PHASE MODULATION}

In this section, we describe the impact of an additional phase modulation of the pulse on the spectral compression performances in the pure SPM case. We can see in Fig. 2(a) that at the propagation distance $z_{1}$ the chirp of the pulse is zero near the pulse centre. At the distance $z_{3}$, the chirp features strong oscillations with a close-to-sinusoidal variation in the central region of the pulse. This observation forms the basis of the simple idea proposed here to offset the chirp: impart to the pulse a corrective sinusoidal temporal phase modulation with suitably chosen frequency and amplitude and opposite sign to the chirp to compensate for.
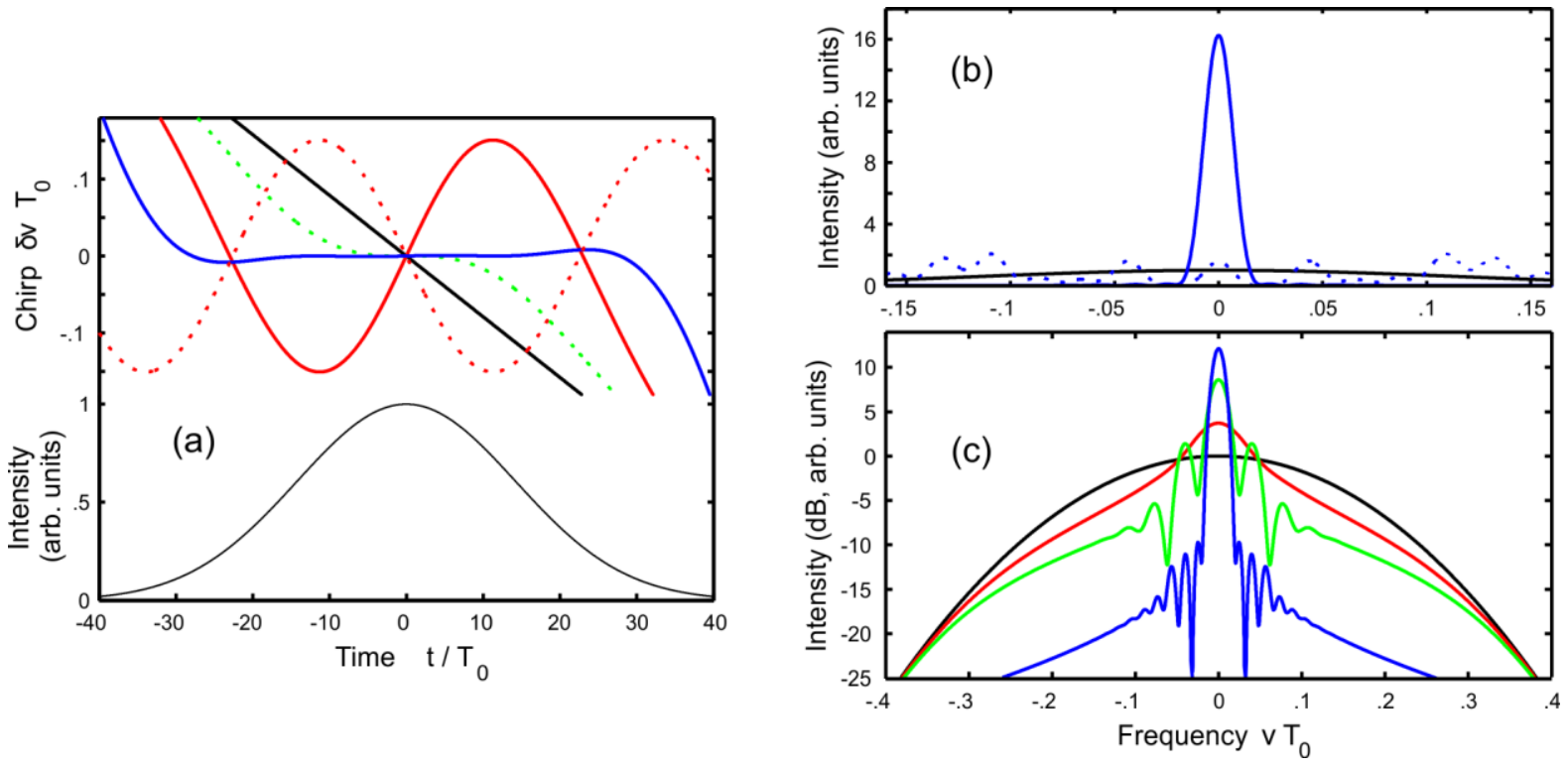

Figure 2. Impact of phase correction on the spectral pulse compression in a purely nonlinear fibre. (a) Temporal chirp profiles at: $z=0$ (black), $z=z_{1}$ (green), and $z=z_{3}$ before (solid red) and after (blue) phase correction. Also shown are the corrective phase modulation applied at $z_{3}$ (dashed red), and the initial temporal intensity profile.

(b),(c) Spectral intensity profiles plotted on linear and logarithmic scales at: $z=0$ (black), $z=z_{1}($ red $), z=z_{2}$ (green), and $z=z_{3}$ before (dashed blue) and after (solid blue) phase correction. 
By use of this method, the chirp is indeed made linear and flat over most of the pulse. The resulting spectrum [Fig. 2(b)] highlights the significant enhancement of the spectral compression afforded by the corrective approach: most of the pulse energy is concentrated in the central lobe of the spectrum while the intensity level of the side lobes is strikingly lower, which entails a significant increase in spectral brilliance.

Figure 3 summarizes the results obtained using the corrective phase modulation method. The strength of the approach increases with increasing propagation distance in the fibre beyond $z_{2}$ as the pulse chirp develops a closer-to-sinusoidal temporal variation. The compression ratio is significantly increased with this strategy both in terms of FWHM and rms widths. Remarkably, the rms compression factor and the Strehl ratio feature up to threefold and twofold increase, respectively, with respect to their maximum value in the absence of phase correction, confirming the efficient reduction of spectral wings and enhanced quality of the compression.

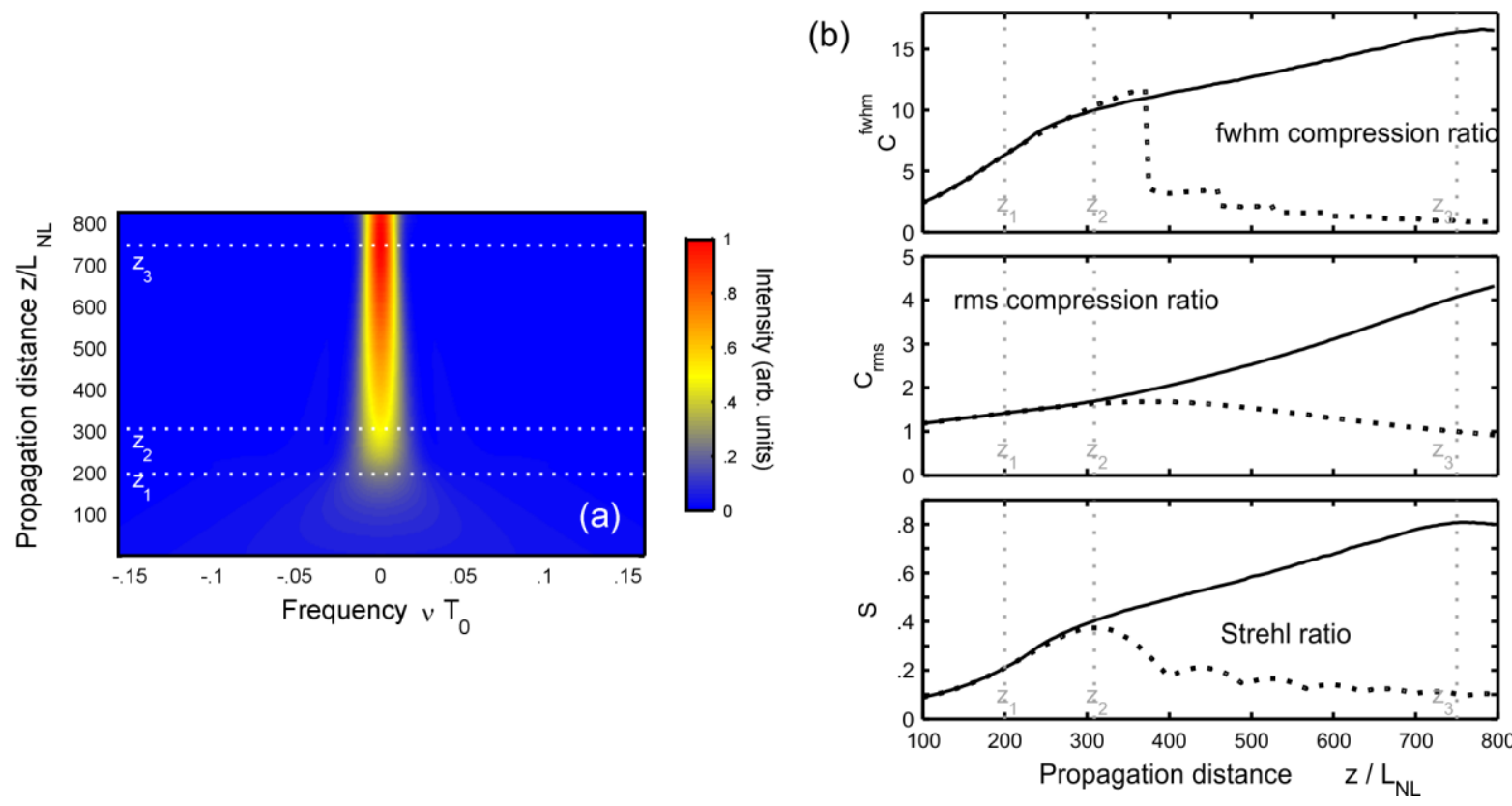

Figure 3. Spectral pulse compression in a purely nonlinear fibre with phase correction: longitudinal evolutions of (a) the spectral intensity profile, and (b) the compression ratios $C_{f w h m}, C_{r m s}$ and the Strehl ratio $S$ (solid). Also shown are the results obtained without correction (dashed).

\section{EVOLUTION IN A NORMALLY DISPERSIVE FIBRE}

The performance characteristics of the spectral compression process can also be enhanced by taking advantage of the progressive reshaping of the initial pulse that occurs upon propagation in a highly nonlinear fibre with normal dispersion. We have performed a detailed analysis of the spectral compression process taking into account the influence of GVD, based on numerical simulation of the nonlinear Schrödinger equation propagation model. In this context, for a given value of the initial stretching factor, finding the best system parameters results in a two-dimensional optimization problem in the plane $(\xi, N)$, where $\xi=z / L_{D}$, parameter $N$ is defined as $N^{2}=L_{N L} / L_{D}$, and $L_{D}=T_{0}^{2} / \beta_{2}$ is the dispersion length $\left(\beta_{2}\right.$ is the fibre GVD coefficient) associated with the pulse at the input to the linear element. The results obtained for an initial stretching factor of 20 are summarized in Fig. 4(a). Optimum compression occurs at the point $\left(\xi_{o p t}=13.6, N_{o p t}=4.4\right)$, and the Strehl ratio is about 0.9 at this point. The shape of the resulting spectrum [Fig. 4(b)] indicates that the pulse is very close to the Fouriertransform limit. The GVD significantly changes the evolution of the pulse spectrum in the fibre [Fig. 4(c)]: contrary to the pure SPM case [Fig. 1(a)], the spectrum does not split into multiple sidebands after the stage of optimum compression. The concomitant temporal dynamics are illustrated in Fig. 4(d): the combination of SPM and GVD make the initial Gaussian pulse reshape into an almost rectangular pulse form at the point of optimum compression, while nearly complete compensation of the pulse chirp occurs. The nearly rectangular pulse shape explains the sine-cardinal-like shape of the spectrum. Figure 4(d) also shows that the pulse acquires an almost parabolic intensity profile before the point of optimum compression (at $\xi=9$ ). Contrary to the parabolic pulses that can be generated through progressive reshaping of initially un-chirped pulses upon nonlinear propagation in a normally dispersive fibre [8], the pulse formed in the parameter region of optimum spectral compression features a very low chirp value over its central part. 

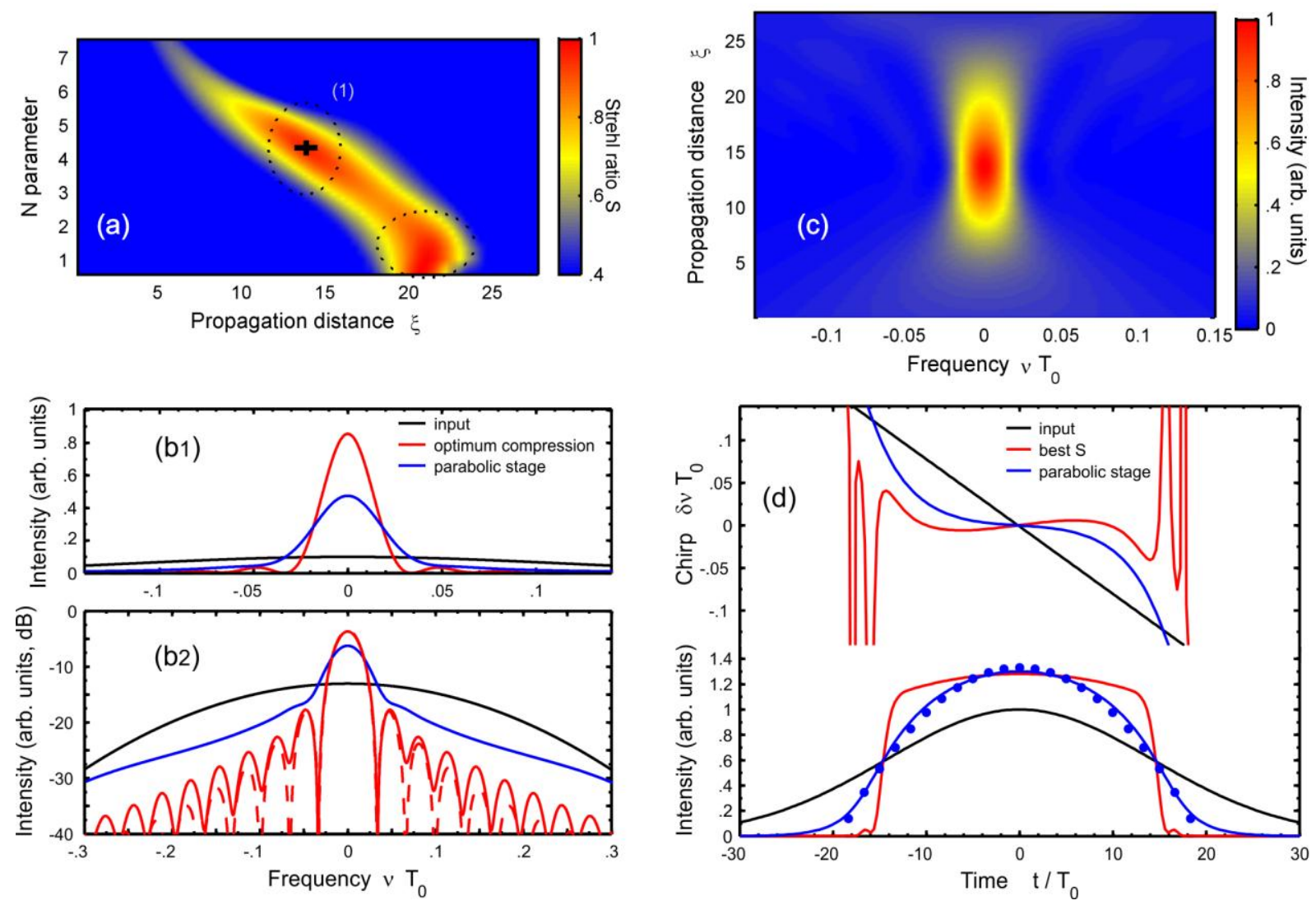

Figure 4. Spectral pulse compression in a nonlinear dispersive fibre. (a) Evolution of the Strehl ratio versus $\xi$ and $N$. (b) Spectral intensity profiles of a pulse with the optimum power parameter $N_{\text {opt }}=4.4$ at different distances in the fibre plotted on linear and logarithmic scales. The spectrum the entrance of the fibre (black) is compared with the spectrum at the distance of optimum compression $\xi_{\text {opt }}=13.6$ (solid red), and the Fouriertransform-limited spectrum expected at this distance (dashed red). Also shown is the spectrum at the distance $\xi=9$ where the temporal profile is close to a parabola (blue). (c) Longitudinal evolution of the spectral intensity profile for $N=N_{\text {opt }}$ (d) Temporal intensity and chirp profiles at different stages of propagation (same colors as subplot b1). The intensity profile at $\xi=9$ is compared to a parabolic fit (blue circles).

\section{CONCLUSIONS}

We have presented two new approaches to enhance the spectral compression of negatively chirped pulses that occurs upon nonlinear propagation in a fibre. In the purely nonlinear regime of propagation, the intensity level of the spectral side lobes can be efficiently reduced by an additional sinusoidal temporal phase modulation imparted to the pulse. Another strategy is to operate in a propagation regime in which normal dispersion reshapes the initial stretched pulse to a near-Fourier-transform-limited rectangular waveform.

\section{REFERENCES}

[1] R. H. Stolen and C. Lin, "Self-phase modulation in silica optical fibers," Phys. Rev. A, vol. 17, pp. 1448-1453, 4/1978 1978.

[2] A. V. Zohrabian and L. K. Mouradian, "Compression of the spectrum of picosecond ultrashort pulses," Quantum Electronics, vol. 25, p. 1076, 1995.

[3] M. Oberthaler and R. A. Höpfel, "Spectral narrowing of ultrashort laser pulses by self-phase modulation in optical fibers," Appl. Phys. Lett., vol. 63, pp. 1017-1019, 19931993.

[4] B. R. Washburn, et al., "Transform-limited spectral compression due to self-phase modulation in fibers," Opt. Lett., vol. 25, pp. 445-447, 4/2000 2000.

[5] J. Fatome, et al., "All-fiber spectral compression of picosecond pulses at telecommunication wavelength enhanced by amplitude shaping," Appl. Opt., vol. 51, pp. 4547-4553, 2012.

[6] A. A. Kutuzyan, et al., "Dispersive regime of spectral compression," Quantum Electronics, vol. 38, pp. 383-387, 2008.

[7] C. Finot and S. Boscolo, "Design rules for nonlinear spectral compression in optical fibers," J. Opt. Soc. Am. B, vol. 33, pp. 760-767, 2016.

[8] C. Finot, et al., "Parabolic pulse generation through passive nonlinear pulse reshaping in a normally dispersive two segment fiber device," Opt. Express, vol. 15, pp. 852-864, 2007. 